\title{
Expression of tissue-specific imprinted gene tumor Suppressing Subtransferable Candidate 4 (TSSC4) is altered in placentae produced by nuclear transfer in cattle
}

\author{
João C.T. Penteado ${ }^{\mathrm{a}, 1}$, Rodolpho J. Borduchi ${ }^{\mathrm{a}, 1}$, Mariângela B.C. Maldonado ${ }^{\mathrm{a}}$, \\ Juliano R. Sangallib ${ }^{\mathrm{b}}$, Tiago H.C. de Bem ${ }^{\mathrm{b}}$, Flavio V. Meirelles ${ }^{\mathrm{b}}$, Daniel R. Arnold ${ }^{\mathrm{c}}$, \\ Flavia L. Lopes ${ }^{\text {a,* }}$ \\ a São Paulo State University (Unesp), School of Veterinary Medicine, Araçatuba, São Paulo, Brasil, 16050-680, Brazil \\ ${ }^{\mathrm{b}}$ University of São Paulo (USP), Faculty of Animal Science and Food Engineering (FZEA), 13635-000 Pirassununga, SP, Brazil \\ c In Vitro Brasil S/A, Mogi Mirim, São Paulo, 13800-970, Brazil
}

\section{A R T I C L E I N F O}

\section{Keywords:}

Bovine

Placenta

TSSC4

H3K4me2

H3K9me2

\begin{abstract}
A B S T R A C T
Embryonic and placental development is highly orchestrated by epigenetic processes. Disruptions in normal placental development, commonly observed in pregnancies produced by nuclear transfer, are associated with abnormal gene expression and altered epigenetic regulation of imprinted and vital placental genes. The objective of this study was to evaluate expression and epigenetic regulation of the imprinted gene TSSC4 in cotyledonary and intercotyledonary tissues from day 60 pregnancies produced by embryo transfer (ET), in vitro fertilization (IVF) and nuclear transfer (NT) in cattle. TSSC 4 expression was reduced by $30 \%$ in cotyledons at 60 days of gestation in the NT group. The proximal promoter region of TSSC4 showed an increase in the permissive histone mark (H3K4me2) and a reduction in the inhibitory histone mark (H3K9me2) in the cotyledons produced by NT, in relation to cotyledons produced by embryo transfer. Interestingly, H3K9me2 was also significantly reduced in cotyledons produced by IVF, compared to the ET controls. DNA methylation, in CpG-rich regions located at the proximal promoter region and the coding region of TSSC4 did not differ. These results suggest that the reduction in TSSC4 expression, observed following NT, can not be explained by the histone changes investigated in the proximal promoter region of the gene, or by changes in methylation in three regions evaluated. Also, a decrease in the levels of H3K9 dimethylation in IVF samples, indicate that in vitro culturing could corroborate with the alterations seen in the NT group.
\end{abstract}

\section{Introduction}

Gene regulation of placental development is vital for successful pregnancy. The precision of when, where and how much a particular transcript is expressed is heavily dependent on epigenetics. Epigenetic regulation of placental development has been well documented, with 75 imprinted genes identified in mouse placenta (Tunster et al., 2013). Genomic imprinting is caused by epigenetic mechanisms such as DNA methylation, histone modifications and non-coding RNA, alone or in various combinations (Monk, 2015). One important example of epigenetic control of placental specific genes is in the CDKN1C/KCNQ1OT1 imprinted cluster region.

\footnotetext{
* Corresponding author.

E-mail address: flavialopes@fmva.unesp.br (F.L. Lopes).

${ }^{1}$ Authors contributed equally to this work.
} 
CDKN1C/KCNQ1OT1 imprinted gene cluster is located on the distal end of mouse chromosome 7, human chromosome 11p15.5 and bovine chromosome 29 (Wang et al., 2015), and is about one megabase in length, containing 10-12 imprinted genes (Kanduri 2016). In the mouse, this region contains up to 16 imprinted genes involved in extraembryonic or placental development (Bressan et al., 2009). In addition, all forms of epigenetic regulation play a role in this region. KCNQ1OT1, also known as LIT1, is a long noncoding RNA that is transcribed in an antisense orientation in respect to the KCNQ1 gene (Kanduri, 2016). The expression of KCNQ1OT1 is controlled by a DNA methylation region, KvDMR1, only allowing for the expression of the paternal allele (Kanduri, 2016). In mice, it has been demonstrated that Kcnq1ot1 interacts with histone (Ezh2 and G9a) and DNA (Dnmt1) modifiers to help silence both ubiquitously and placental-specific imprinted genes (Redrup et al., 2009; Mohammad et al., 2010)

One gene included in the CDKN1C/KCNQ1OT1 domain is Tumor Suppressing Subtransferable Candidate 4 (TSSC4), a gene that plays a role in malignancies and diseases associated with this region (Paulsen et al., 2000). TSSC4 is expressed in several fetal and adult tissues in cattle (Zaitoun and Khatib, 2008) and humans (Lee et al., 1999). Imprinting of TSSC4 in the placenta of mice (Lewis et al., 2004) and cattle (Zaitoun and Khatib, 2008) has been reported. However, in placental tissues of humans (Lee et al., 1999) and pigs (Jiang et al., 2015), TSSC4 is not imprinted, thus qualifying its process of imprinting as tissue- and species-specific. Little is known about the function of TSSC4 in relation to placental development or which epigenetic events control its placental imprinting.

While most of the information about epigenetic control of placental genes has come from transgenic mouse studies, studies in other species have relied on models of placental abnormalities. These placental problems are most evident in pregnancies produced by assisted reproductive technologies such as nuclear transfer (Arnold et al., 2008; Chavatte-Palmer et al., 2012). While various animals have been cloned successfully, the technique is still inefficient, with a birth rate of healthy calves at around 4-6\% (Campbell et al., 2007). Many of these pregnancies are lost during the first trimester, mostly caused by abnormal placental attachment, development or growth (Hill et al., 2000). In addition, several placental specific genes have shown to be abnormally expressed and their epigenetic regulation altered (Niemann, 2016; Arnold et al., 2017).

The objective of this study was to evaluate the expression of the placental-specific, imprinted gene TSSC4 in cotyledonary and intercotyledonary tissues of day 60 placentas produced by embryo transfer (ET), in vitro fertilization (IVF) and nuclear transfer (NT) in cattle, and also to investigate the epigenetic control of this gene in the bovine placenta produced by the different reproductive biotechnologies.

\section{Material and methods}

All animal protocols were approved by the Ethics Committee on Animal Use from the Universidade Estadual Paulista, in accordance with the regulations of the National Council for Animal Experiments Control (CONCEA).

Cotyledonary and intercotyledonary tissues were collected at 60 days of gestations, produced by ET, IVF and NT, using Bos indicus females (Nelore) and Bos taurus (Hereford) semen, purchased at CRV Lagoa (Sertãozinho, SP, BRAZIL). All three groups were produced and collected as previously described (Arnold et al., 2017). Briefly, for the ET group, embryos were collected 7 days postinsemination, using uterine flushing and transferred into crossbred synchronized recipients.

For the IVF group, the same females used as embryo donors for the ET group, were used as oocyte donors, and these oocytes were in vitro fertilized using the same Hereford semen as in the ET group. Fertilized oocytes were then cultured for 7 days in synthetic oviductal fluid (SOF) medium plus amino acids (1.4 mM glycine, $0.4 \mathrm{mM}$ alanine, $1 \mathrm{mM}$ glutamine, $2 \%$ essential amino acids, $1 \%$ non-essential amino acids), supplemented with $2.5 \% \mathrm{FBS}$ and $5 \mathrm{mg} / \mathrm{mL}$ fatty acid-free bovine serum albumin (FAF-BSA), at $38{ }^{\circ} \mathrm{C}$ in a humidified atmosphere of 5\% CO2 and 5\% O2.

For the production of the NT group, fetal fibroblast cells were collected from seven of the 60 day old fetuses produced for the IVF group, thus eliminating the genotypic variability. A fetal fibroblast cell was introduced into the perivitelline space of matured, enucleated oocyte that were matured for $24 \mathrm{~h}$ (presence of 1st polar body). The couplet was then electro-fused (1.5-kV electric pulse lasting $70 \mathrm{~ms}$ ) in a solution of $0.3 \mathrm{M}$ mannitol, $0.1 \mathrm{mM} \mathrm{MgSO} 4$ and $0.05 \mathrm{mM} \mathrm{CaCl2}$. Fused couplets underwent parthenogenetic activation by exposing to $5 \mathrm{mM}$ ionomycin for $4 \mathrm{~min}$ and $2 \mathrm{mM}$ dimethylaminopurine (6-DMAP) for $3 \mathrm{~h}$. Reconstructed oocytes were cultured for 7 days in SOF media, similar to IVF embryos. On day 7, both IVF and NT embryos were transferred into crossbred synchronized recipients.

\subsection{Sample collection}

One to two days prior to slaughter, rectal ultrasound was performed to confirmed viable pregnancies in all groups (ET, IVF and NT), and on day 60 of gestation the animals were slaughtered. The pregnancy rate for the ET group was 75\% (3/4), 33.3\% (6/18) for IVF, and 19.4\% (7/36) for NT. Gravid uteri were transported on ice to the laboratory to be processed, within an hour from slaughter. Cotyledonary (pools of 3-4 per placenta) and intercotyledonary (pools of 2-3 separate areas per placenta) tissues were collected and frozen in liquid nitrogen and stored at $-80{ }^{\circ} \mathrm{C}$ until analysis.

\subsection{Real-time quantitative polymerase chain reaction ( $q P C R$ )}

Samples collected from cotyledonary and intercotyledonay tissues from ET ( $n=3$ pregnancies), IVF ( $n=6$ pregnancies) and NT ( $n=7$ pregnancies) groups were used for analysis of gene expression by qPCR. RNA was extracted using the RNeasy Mini kit (Qiagen, Mississauga, Canada), reverse transcribed ( $1.5 \mu \mathrm{g}$ of total RNA/sample) using the Moloney murine leukaemia virus (MML-V) enzyme (Life Technologies) and oligo-dT primers. PCR was performed using a Quantitect SYBR green kit (Qiagen) in an MxPRO3005 real- 
Table 1

Sequence of initiators used for the analysis of polymerase chain reaction (PCR) and Chromatin Immunoprecipitation.

\begin{tabular}{lll}
\hline Gene & Forward $5^{\prime}-3^{\prime}$ & Reverse 5'-3' \\
\hline GAPDH & AAGGCCATCACCATCTTCCA & CCACTACATACTCAGCACCAGCAT \\
TSSC4 & AAGATGTGTCCCAAGGTTGC - ChIP & AGACCGGAATTGACTCAGGA \\
& AAGATGTGTGTCCCAAGGTTGC & GGATTGAACCCAGGTCTCCT \\
\hline
\end{tabular}

time PCR machine (Agilent, Santa Clara, CA, USA). Standard curves were used for all genes at each individual run, and the expression of candidate genes is presented as a ratio to the housekeeping control, which was chosen on previous comparative analysis on these samples, between three housekeeping genes. All primers were designed to span at least one intron, to avoid repeat regions and similarities to other non-specific genomic regions. The bovine sequence, available on the University of California, Santa Cruz (UCSC) Genome Browser, was employed for primer design, using the Primer3 program (Rozen and Skaletsky, 2000). Primers are described in Table 1.

\subsection{Chromatin immunoprecipitation (ChIP)}

ChIP was performed as previously described (Arnold et al., 2017) to study the presence of histone modifications (permissive: H3K4me2 and inhibitory: H3K9me2) on the proximal promoter region of TSSC4 in the cotyledonary tissues of the three experimental groups. The kit Simple Enzymatic ChIP Chromatin IP (Cell Signaling Technology, Danvers, MA, USA) was used, following the manufacturer's instructions. Approximately $50 \mathrm{mg}$ of cotyledonary tissues were minced and incubated with $1.5 \%$ formaldehyde in PBS containing a protease inhibitor cocktail for $20 \mathrm{~min}$ at room temperature. After crosslinking, tissues were washed in PBS + PIC and broken with a dounce homogenizer. After homogenization, samples were washed with PBS and chromatin was sheared by sonication (eight pulses of $10 \mathrm{~s}$ at $50 \%$ amplitude with $1 \mathrm{~min}$ intervals between pulses), using a sonicator Vibracell sonicator (Sonics and Materials, Newtown, CT, USA). Anti-dimethyl H3K9 (dilution 1:25, EMD Millipore, Temecula, CA, USA), anti-dimethyl H3K4 (1:25 dilution, Cell Signaling Technology) or non-immune rabbit IgG (used as negative control) were used to precipitate the sonicated chromatin, and the samples were immunoprecipitated at $4{ }^{\circ} \mathrm{C}$ overnight. Protein G-agarose beads were employed to recover the precipitated DNA, which was then purified on columns supplied by purification kit, following reversal of cross-linking. Primers used for real time PCR are presented in Table 1, and results are shown as a ratio of precipitated DNA over input control (total nonprecipitated DNA).

\subsection{Evaluation of quantitative DNA methylation by real-time PCR ( $q A M P$ )}

Cotyledons of all three experimental groups (ET, IVF and NT), collected at 60 days of gestation, were used for quantitative DNA methylation analysis evaluated by qAMP (Oakes et al., 2009). Cotyledonary DNA (1 $\mu$ g/enzyme) was digested with methylationsensitive restriction enzymes (HhaI, New England Biolabs, County Rd, Ipswich, USA); (HpaII, Carlsbad, California), both cutting unmethylated DNA, and a methylation-dependent enzyme (McrBC, New England Biolabs, County Road, Ipswich, USA), which cleaves methylated DNA. Digested and undigested DNA was used for real-time PCR reactions using the Quantitect SYBR Green kit (Qiagen, Mississauga, ON, Canada), following the manufactureŕs recommendation. Methylation is shown as a percentage of digested over undigested DNA, based on the shift in Ct obtained for each primer pair. All primers were designed using the Primer3 program, based on the bovine sequence available on the University of California, Santa Cruz (UCSC) Genome Browser, avoiding repeat regions and similarities to other non-specific genomic regions. Regions were chosen to contain recognition sites to all three enzymes employed. Primer sequences are depicted in Table 2.

\subsection{Statistical analysis of results}

Gene expression, ChIP and digestion results were analyzed using least-squares analysis of variance and the general linear model procedures of SAS (SAS Institute, Cary, NC, USA). Comparison of means was done using Duncan's multiple range test, and significance was set at $\mathrm{p}<0.05$.

Table 2

Sequencing primers used for quantitative analysis of methylation (qAMP).

\begin{tabular}{|c|c|c|}
\hline Oligo name & Sequence $\left(5^{\prime}-3^{\prime}\right)$ & Position of product on UMD_3.1.1/bosTau8 \\
\hline TSSC4 qbody A (Forward) & GCTGTGCCTGCTGTGACTCC & chr29:49837091-49837340 \\
\hline TSSC4 qbody 1 (Reverse) & TCTGCGGCCCCAGGAAG & \\
\hline TSSC4 qprom 1-A (Forward) & GGTGCCTTGTGGGAGATGTA & chr29:49839884-49840161 \\
\hline TSSC4 qprom 1-1 (Reverse) & GTGTCAGCTGCCTGAGTCCT & \\
\hline TSSC4 qprom 2-A (Forward) & AGAGACAACCCTCAAGGAAGC & chr29:49839615-49839808 \\
\hline TSSC4 qprom 2-1 (Reverse) & CCGTGATCAGTGGGACACA & \\
\hline
\end{tabular}



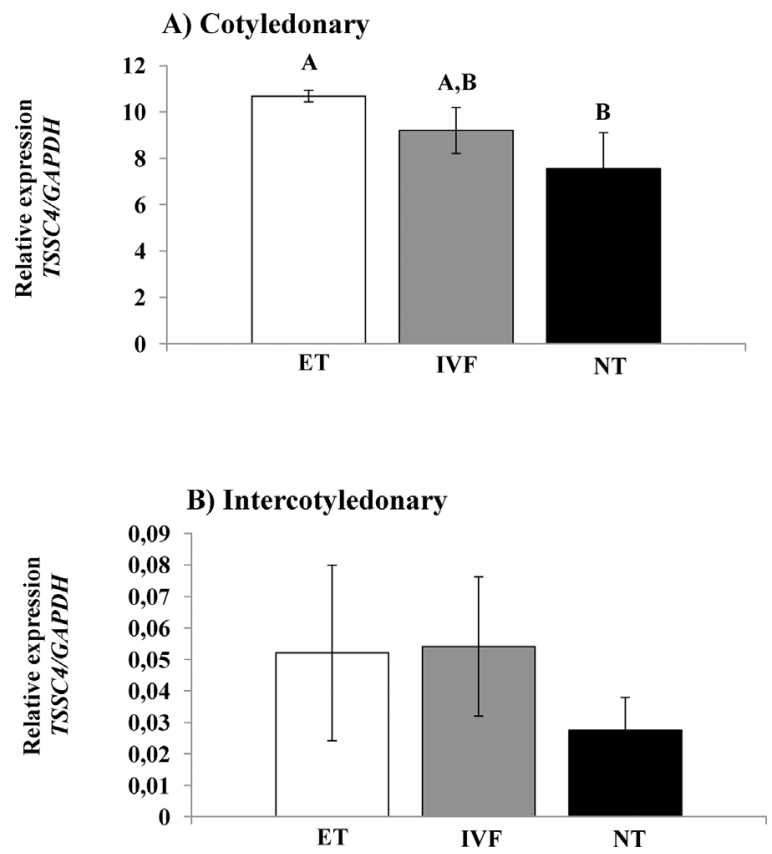

Fig. 1. Expression of TSSC4 in (A) cotyledonary and (B) intercotyledonary tissue samples from ET $(n=3)$, IVF ( $n=6)$ and NT ( $n=7)$ day 60 pregnancies. Cotyldonary samples were pools of 3-4 per placenta. Intercotyledonary samples were pools of 2-3 different areas per placenta. Different superscript letters differ significantly $(\mathrm{p}<0.05)$.

\section{Results}

\subsection{Expression of TSSC4 in ET, IVF and NT placental tissues}

Expression of TSSC4 was decreased by $30 \%$ in NT cotyledons from day 60 of gestation, compared to control samples produced by ET (Fig. 1A; p < 0.05). Samples produced by IVF showed intermediate values to those observed for the ET and NT groups (Fig. 1A). Expression from intercotyledonary tissues was not significantly different among the groups (Fig. 1B). In addition, intercotyledonary tissue expressed less TSSC4 than its cotyledonary counterpart, regardless of group.

\subsection{Association of di-methylated H3K4 and H3K9 histones in TSSC4 promoter region}

Methylation of H3K4 and H3K9 associated with the proximal promoter of the TSSC4 gene was altered by the use of assisted reproductive techniques. Chip analysis for the permissive histone mark H3K4me2 in cotyledonary tissues showed significantly higher dimethylation levels in NT samples, compared to samples produced by ET and IVF (Fig. 2A). Contrary to H3K4 results, analysis of the repressive histone mark H3K9me2 was significantly lower in NT samples compared to ET samples (Fig. 2B). Interestingly, H3K9me2 levels in IVF samples were similar to those observed for the NT group, also significantly differing from the ET group (Fig. 2B).

\subsection{DNA methylation of TSSC4}

Quantitative analysis of DNA methylation using real-time PCR (qAMP) technique was utilized to assess methylation in genic regions of TSSC4 in day 60 cotyledonary tissues from ET, IVF and NT gestations. Two separate regions within the proximal promoter (CpG rich regions: prom 1 at -2236 and prom 2 at -1968 from the ATG triplet), and one region within the coding sequence (body) of the imprinted gene TSSC4 were analyzed. No differences in methylation levels between experimental groups were found for the promoter region 1 and 2, which were largely devoid of methylation for all three groups (Fig. 3A and B). Similarly, no differences were found in the gene body of TSSC4 between the groups (Fig. 3C). Methylation levels were numerically higher in the gene body than those found in the promoter regions.

\section{Discussion}

Assisted reproductive techniques in cattle are commonly associated with altered gene expression, abnormal placental/fetal development and increased pregnancy losses. These problems are most evident in embryos produced by nuclear transfer. In addition, the most dramatic changes are in expression of imprinted genes that control fetal and/or placental development. The present study demonstrated reduced expression of TSSC4 in cotyledonary tissues of embryos produced by NT, compared to embryos produced by 

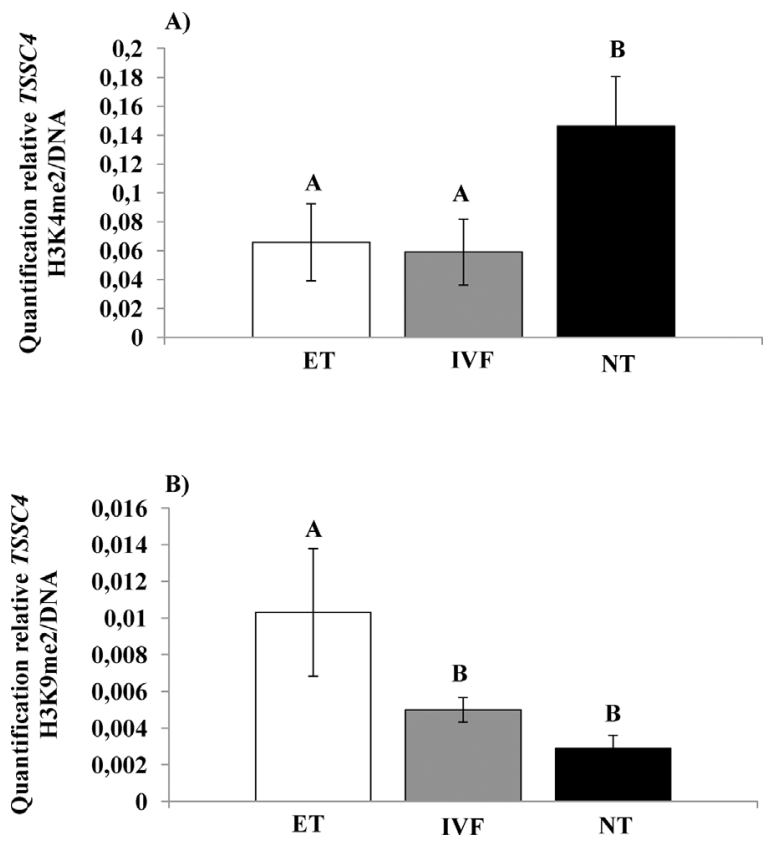

Fig. 2. Chromatin Immunoprecipitacion (ChIP-PCR) for (A) H3K4me2 and (B) H3K9me2 at the proximal promoter region of the gene in TSSC4 in cotyledonary tissue samples of ET $(n=3)$, IVF $(n=6)$ and NT $(n=7)$ collected at day 60 of pregnancy. Cotyldonary samples were pools of 3-4 per placenta. Different superscript letters differ significantly $(\mathrm{p}<0.05)$.

artificial insemination/embryo transfer. In addition, histone modifications (H3K4me2 and H3K9me2) in the promoter region of TSSC4 were altered in NT cotyledonary tissues in a contrary pattern to gene expression. DNA methylation in three regions of TSSC4 did not vary between the experimental groups.

TSSC4 is located within the KCNQ1 imprinted domain on chromosome 29 in cattle (Khatib et al., 2007). Several of the imprinted genes in this region play a role in placental development in cattle (Khatib et al., 2007), mice (Lewis et al., 2004) and human (Monk 2015; Khatib et al., 2007). More similar to the mouse than human, TSSC4 in the cow is only imprinted in placental tissues (Zaitoun and Khatib 2008; Wang et al., 2015). To date, the primary function of TSSC4 and its role in placental development remain unknown. However, it may play a role in the malignancies and diseases that are associated with this genetic region. Similar to the current study, altered expression of other placental-specific imprinted genes in NT placental tissues have been documented (Jouneau and Renard, 2003; Smith et al., 2005; Arnold et al., 2006; Chavatte-Palmer et al., 2012; Salilew-Wondim et al., 2013; Arnold et al., 2017). While TSSC4 expression in the intercotyledonary tissue was not different between treatments, it appeared to follow a similar pattern, with NT samples having the lowest levels. The difference in expression between the tissues, regardless of group, showed cotyledonary expression 200-300 fold greater than intercotyledonary. This may suggest a role of TSSC4 in cotyledonary formation, in response to caruncular tissue of the uterus. Further studies are needed to determine which factors and which cells may be stimulated to increase TSSC4 expression.

In an in vitro production system, TSSC4 expression was reported to be increased in degenerate embryos when compared to normal blastocysts (Driver et al., 2013), suggesting proper regulation of TSSC4 is important for early embryo development. Our current study showed lower TSSC4 expression in NT samples, compared to ET. These results may suggest a temporal regulation in expression patterns, or yet that a balance in expression may influence proper embryo/fetal development. As it is expected, a loss of imprinting would upregulate gene expression. However, our results demonstrated a reduction in TSSC4 expression at day 60, therefore allelic contribution was not further investigated. It must be noted that the morphological analysis of fetal and placental samples used in this study were previously reported (Arnold et al., 2017). Those results demonstrated slightly larger fetuses in the IVF and NT groups, but no differences in numbers of cotyledons were observed, compared to the ET group. However, there were a lower percentage of binucleate cells in the NT group, followed by IVF, with greatest percentage in the ET control group (Arnold et al., 2017).

To determine if altered epigenetic events could explain the difference in gene expression, both histone modification and DNA methylation were investigated. Histone modifications were chosen based on work in the mouse which demonstrated H3K4me2 and H3K9me2 associated with the promoter region of Tssc4 (Lewis et al., 2004; Umlauf et al., 2004). Utilizing ChIP-qPCR, it was demonstrated that both histone modifications were associated with chromatin within $700 \mathrm{bp}$ of the transcription start site. However, the results were in opposition to the gene expression results, with the inhibitory mark (H3K9me2) being higher and the permissive mark (H3K4me2) being lower in the NT samples. It is plausible that either these particular histone modifications or this particular promoter region are not associated with the epigenetic regulation of bovine TSSC4. However, these results demonstrate altered histones modifications due to NT. Interestingly, the inhibitory histone mark H3K9me2 was altered in IVF samples to similar levels as NT samples. This result suggests that in vitro culture is also affecting histone regulation, which has been reported previously (Gaspar 


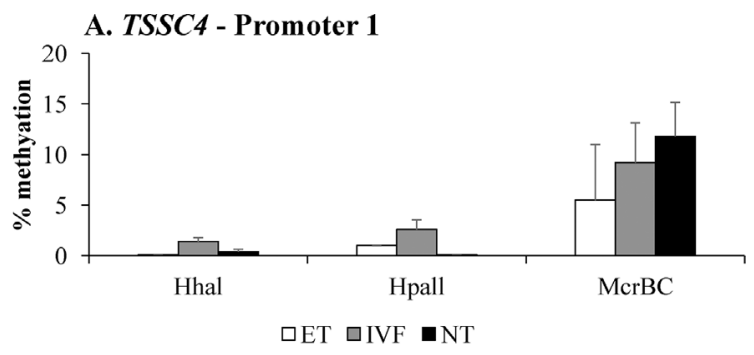

\section{B. TSSC4 - Promoter 2}
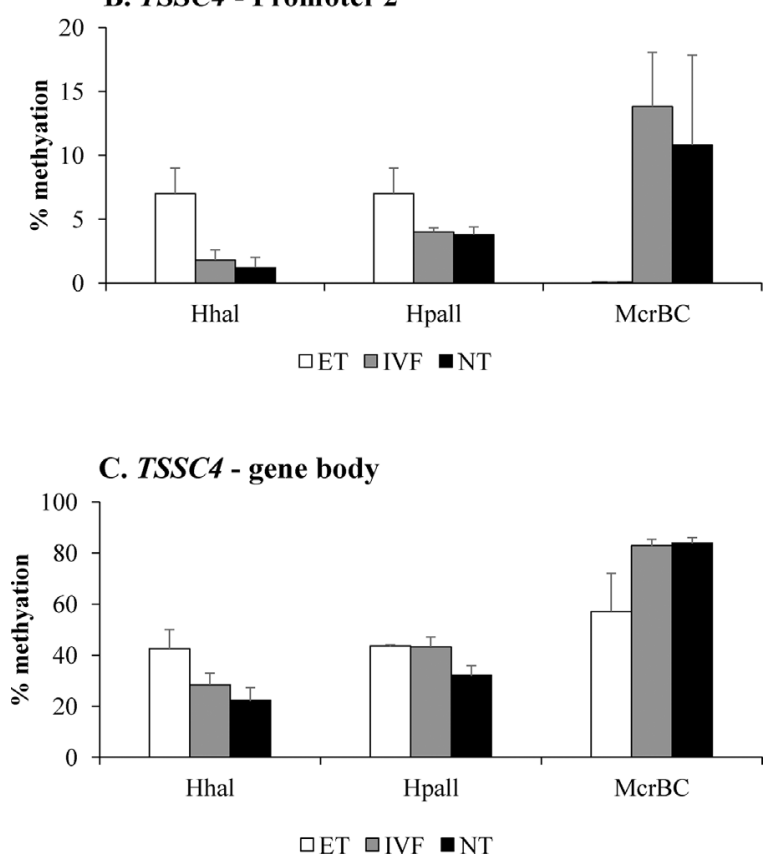

Fig. 3. Quantitative analysis of DNA methylation (qAMP), employing two methylation-sensitive (HpaII and HhaI) and one methylation-dependent enzyme (McrBC), at two CpG rich regions at the proximal promoter (A and B), and one at the body $(\mathrm{C}$ ) of the gene in TSSC4 in cotyledonary tissue samples of ET ( $\mathrm{n}=3$ ), IVF ( $=6$ ) and NT $(n=7)$ collected at day 60 of pregnancy. Cotyledonary samples were pools of 3-4 per placenta.

et al., 2015).

In addition, DNA methylation was investigated in three regions (two promoter and one coding region) of TSSC4. To date, no imprinting control regions (ICR) for TSSC4 have been identified. However, by in silico analysis for CpG islands, these regions were selected. No difference in DNA methylation was identified in the three regions for any of the groups. Interestingly, the coding region had more DNA methylation than both promoter regions evaluated. This either suggests that TSSC4 is not regulated directly by DNA methylation, or these particular regions are not ICRs for TSSC4. Further studies are required to investigate other regions as potential ICRs, as well as other potential epigenetic regulators, such as ncRNAs.

The current study has demonstrated altered TSSC4 gene expression in placental tissues from NT embryos. While histone modifications, H3K4me2 and H3K9me2, were altered between NT and ET samples, these results were contrary to the expression data. In addition, it does not appear that DNA methylation in the three regions investigated control TSSC4 expression. The results from IVF placental samples provided further evidence that in vitro culture also perturbs normal gene regulation/expression and potential embryo development. By understanding the events of placental development, we may be able to improve the results from assisted reproductive techniques.

\section{Conflicts of interest}

None.

\section{Acknowledgments}

Funding for this project was supported by the São Paulo Research Foundation (FAPESP), Brazil (2009/50381-5 for Lopes FL). R. 
Junqueira and M.B.C. Maldonado had scholarships funded by FAPESP during this study. J.C.T. Penteado held a scholarship funded by the Coordination for the Improvement of Higher Education Personnel (Capes), Brazil.

\section{References}

Arnold, D.R., Bordignon, V., Lefebvre, R., Murphy, B.D., Smith, L.C., 2006. Somatic cell nuclear transfer alters peri-implantation trophoblast differentiation in bovine embryos. Reproduction 132, 279-290.

Arnold, D.R., Fortier, A.L., Lefebvre, R., Miglino, M.A., Pfarrer, C., Smith, L.C., 2008. Placental insufficiencies in cloned animals-a workshop report. Placenta 29 (Suppl A), S108-S110.

Arnold, D.R., Gaspar, R.C., da Rocha, C.V., Sangalli, J.R., de Bem, T.H., Correa, C.A., Penteado, J.C., Meirelles, F.V., Lopes, F.L., 2017. Nuclear transfer alters placental gene expression and associated histone modifications of the placental-specific imprinted gene pleckstrin homology-like domain family A, member 2 (PHLDA2) in cattle. Reprod. Fertil. Dev. 29, 458-467.

Bressan, F.F., De Bem, T.H., Perecin, F., Lopes, F.L., Ambrosio, C.E., Meirelles, F.V., Miglino, M.A., 2009. Unearthing the roles of imprinted genes in the placenta. Placenta 30, 823-834.

Campbell, K.H., Fisher, P., Chen, W.C., Choi, I., Kelly, R.D., Lee, J.H., Xhu, J., 2007. Somatic cell nuclear transfer: past, present and future perspectives. Theriogenology 68 (Suppl 1), S214-S231.

Chavatte-Palmer, P., Camous, S., Jammes, H., Le Cleac'h, N., Guillomot, M., Lee, R.S., 2012. Review: placental perturbations induce the developmental abnormalities often observed in bovine somatic cell nuclear transfer. Placenta 33 (Suppl), S99-S104.

Driver, A.M., Huang, W., Kropp, J., Penagaricano, F., Khatib, H., 2013. Knockdown of CDKN1C (p57(kip2)) and PHLDA2 results in developmental changes in bovine pre-implantation embryos. PLoS One 8, e69490.

Gaspar, R.C., Arnold, D.R., Correa, C.A., da Rocha Jr., C.V., Penteado, J.C., Del Collado, M., Vantini, R., Garcia, J.M., Lopes, F.L., 2015. Oxygen tension affects histone remodeling of in vitro-produced embryos in a bovine model. Theriogenology 83, 1408-1415.

Hill, J.R., Burghardt, R.C., Jones, K., Long, C.R., Looney, C.R., Shin, T., Spencer, T.E., Thompson, J.A., Winger, Q.A., Westhusin, M.E., 2000. Evidence for placental abnormality as the major cause of mortality in first-trimester somatic cell cloned bovine fetuses. Biol. Reprod. 63, $1787-1794$.

Jiang, Z., Dong, H., Zheng, X., Marjani, S.L., Donovan, D.M., Chen, J., Tian, X.C., 2015. mRNA levels of imprinted genes in bovine In vivo oocytes, embryos and cross species comparisons with humans, mice and pigs. Sci. Rep. 5, 17898.

Jouneau, A., Renard, J.P., 2003. Reprogramming in nuclear transfer. Curr. Opin. Genet. Dev. 13, 486-491.

Kanduri, C., 2016. Long noncoding RNAs: lessons from genomic imprinting. Biochim. Biophys. Acta. 1859, $102-111$.

Khatib, H., Zaitoun, I., Kim, E.S., 2007. Comparative analysis of sequence characteristics of imprinted genes in human, mouse, and cattle. Mamm. Genome 18, $538-547$.

Lee, M.P., Brandenburg, S., Landes, G.M., Adams, M., Miller, G., Feinberg, A.P., 1999. Two novel genes in the center of the 11 p15 imprinted domain escape genomic imprinting. Hum. Mol. Genet. 8, 683-690.

Lewis, A., Mitsuya, K., Umlauf, D., Smith, P., Dean, W., Walter, J., Higgins, M., Feil, R., Reik, W., 2004. Imprinting on distal chromosome 7 in the placenta involves repressive histone methylation independent of DNA methylation. Nat. Genet. 36, 1291-1295.

Mohammad, F., Mondal, T., Guseva, N., Pandey, G.K., Kanduri, C., 2010. Kcnq1ot1 noncoding RNA mediates transcriptional gene silencing by interacting with Dnmt1. Development 137, 2493-2499.

Monk, D., 2015. Genomic imprinting in the human placenta. Am. J. Obstet. Gynecol. 213 (4 Suppl), S152-S162.

Niemann, H., 2016. Epigenetic reprogramming in mammalian species after SCNT-based cloning. Theriogenology 86, 80-90.

Oakes, C.C., La Salle, S., Trasler, J.M., Robaire, B., 2009. Restriction digestion and real-time PCR (qAMP). Methods Mol. Biol. 507, 271-280.

Paulsen, M., El-Maarri, O., Engemann, S., Strodicke, M., Franck, O., Davies, K., Reinhardt, R., Reik, W., Walter, J., 2000. Sequence conservation and variability of imprinting in the Beckwith-Wiedemann syndrome gene cluster in human and mouse. Hum. Mol. Genet. 9, $1829-1841$.

Redrup, L., Branco, M.R., Perdeaux, E.R., Krueger, C., Lewis, A., Santos, F., Nagano, T., Cobb, B.S., Fraser, P., Reik, W., 2009. The long noncoding RNA Kcnq1ot1 organises a lineage-specific nuclear domain for epigenetic gene silencing. Development 136, 525-530.

Rozen, S., Skaletsky, H., 2000. Primer3 on the WWW for general users and for biologist programmers. Methods Mol. Biol. 132, 365-386.

Salilew-Wondim, D., Tesfaye, D., Hossain, M., Held, E., Rings, F., Tholen, E., Looft, C., Cinar, U., Schellander, K., Hoelker, M., 2013. Aberrant placenta gene expression pattern in bovine pregnancies established after transfer of cloned or in vitro produced embryos. Physiol. Genom. 45, 28-46.

Smith, S.L., Everts, R.E., Tian, X.C., Du, F., Sung, L.Y., Rodriguez-Zas, S.L., Jeong, B.S., Renard, J.P., Lewin, H.A., Yang, X., 2005. Global gene expression profiles reveal significant nuclear reprogramming by the blastocyst stage after cloning. Proc. Natl. Acad. Sci. 102, $17582-17587$.

Tunster, S.J., Jensen, A.B., John, R.M., 2013. Imprinted genes in mouse placental development and the regulation of fetal energy stores. Reproduction 145, R117-R137.

Umlauf, D., Goto, Y., Cao, R., Cerqueira, F., Wagschal, A., Zhang, Y., Feil, R., 2004. Imprinting along the Kcnq1 domain on mouse chromosome 7 involves repressive histone methylation and recruitment of Polycomb group complexes. Nat. Genet. 36, 1296-1300.

Wang, M., Li, D., Zhang, M., Yang, W., Wu, G., Cui, Y., Li, S., 2015. Biallelic expression of Tssc4, Nap114, Phlda2 and Osbpl5 in adult cattle. J. Genet. 94, 391-395.

Zaitoun, I., Khatib, H., 2008. Comparative genomic imprinting and expression analysis of six cattle genes. J. Anim. Sci. 86, 25-32. 